\title{
IDIOPATHIC INFANTILE NYSTAGMUS, WITH AND WITHOUT FRMD7 GENE MUTATIONS
}

Clinical features and eye movement recordings of 90 subjects with mutations in the gene (FRMD7 group) were compared to 48 without mutations (non-FRMD7 group) but with clinical idiopathic infantile nystagmus (IIN), in a study at University of Leicester, Leicester Royal Infirmary, Leeds General Infirmary, Royal Preston Hospital, Addenbrooks Hospital, Cambridge, UK; Wills Eye Hospital, Philadelphia, USA; and Medical University Graz, Austria. Visual acuity and binocular vision were generally normal in both groups. Prevalence of strabismus was similar and occurred in $7.8 \%$ of mutation and $10 \%$ of nonFRMD7 patients. Anomalous head posture was significantly more frequent in the nonFRMD7 group ( $\mathrm{P}<0.0001)$; moderate $(5-15$ degrees) in $24 \%$ and severe $(>15)$ in $27 \%$ vs $17 \%$ only moderately affected in the FRMD7 group. Amplitude of nystagmus was lower at primary position in the FRMD7 group ( $\mathrm{P}<0.0001)$ compared to non-FRMD7 group ( $\mathrm{P}=0.83$ ). Pendular nystagmus was more frequent in the FRMD7 group $(\mathrm{P}=0.003)$. Obligate female carriers of an FRMD7 mutation were clinically affected in 53\%. Visual acuity of affected females was better than affected males $(\mathrm{P}=0.014)$. FRMD7 is a major cause of $\mathrm{X}$-linked IIN. The findings are helpful in genetic counselling of patients with idiopathic infantile nystagmus. (Thomas S, Proudlock FA, Sarvananthan N, et al. Phenotypical characteristics of idiopathic infantile nystagmus with and without mutations in FRMD7. Brain May 2008;131:1259-1267). (Respond: Prof Irene Gottlob, Ophthalmology Group, University of Leicester, Faculty of Medicine \& Biological Sciences, Robert Kilpatrick Clinical Sciences Building, Leicester Royal Infirmary, PO Box 65, Leicester, LE2 7LX, UK. E-mail: ig15@le.ac.uk).

COMMENT. Idiopathic infantile nystagmus is noted in the first months of life, and diagnosis is dependent on the absence of albinism and congenital night blindness or achromatopsia. Prevalence of nystagmus is estimated at 2.4/1000 (Sarvananthan $\mathrm{N}$ et al. Invest Ophthalmol Vis Sci 2006;47:E-Abstract 2656). Inheritance is X-linked most commonly, and the present authors report multiple mutations in FRMD7 gene localized to chromosome X (NYS1). In addition, autosomal dominant inheritance is described, localized to chromosome 6 , as well as autosomal recessive inheritance. In patients with idiopathic infantile nystagmus, clinical characteristics of those with FRMD7 mutation are similar to a non-FRMD7 mutation group, except abnormal head posture is significantly less frequent and amplitude of nystagmus in the primary position is lower in those with mutations. Most patients with IIN have good visual acuity and stereopsis and strabismus is infrequent, in both those with and without mutations. Mutations in FRMD7 are the major cause of inherited IIN. Cerebellar dysfunction is the most likely cause of nystagmus related to the FRMD7 gene in IIN. (Glasauer S. Ann NY Acad Sci 2003;1004:206-219).

\section{MANAGEMENT OF NEONATAL SEIZURES}

The generally accepted clinical approaches to neonatal seizures were determined by questionnaires completed by pediatric neurologists and neonatologists representing all the pediatric neurology units and all departments of neonatology in Isrrael, and were evaluated at 
Tel Aviv Sourasky Medical Center. Responding 36/55 (65\%) neurologists and 66/112 (59\%) neonatologists chose similar antiepileptic drugs as first line (phenobarbital), second line (phenytoin), and third line (benzodiazepines) treatments. Treatment duration favored by both specialties varied widely from $1-52$ weeks, neurologists tending to recommend longer treatment for seizures secondary to asphyxia or hemorrhage. For intractable neonatal seizures, neurologists favored valproic acid and topiramate, and neonatologists recommended lidocaine and benzodiazepines $(\mathrm{P}=0.0023)$. Continuous $\mathrm{EEG}$ monitoring after asphyxia was used by $70.5 \%$ of neonatologists contrasting with only $40 \%$ of neurologists $(\mathrm{P}=0.013)$. Specialties differed concerning the harmfulness of neonatal seizures: $76 \%$ neurologists $\mathrm{cf}$ $55 \%$ neonatologists answered "Yes" to "Could neonatal seizures harm the brain?" $(\mathrm{P}=0.065)$; $12 \%$ neurologists of $34 \%$ neonatologists answered "Don't know." "Could electrographic seizures harm the brain?;" $43 \%$ neurologists and $47 \%$ neonatologists answered "Don't know." "Would you treat electrographic seizures?," $40 \%$ neurologists and $38 \%$ neonatologists answered "Yes." Controlled clinical trials to establish evidence-based guidelines for the management of neonatal seizures are indicated. (Bassan H, Bental Y, Shany E et al. Neonatal seizures: dilemmas in workup and management. Pediatr Neurol June 2008;38:415-421). (Dr Haim Bassan, Neonatal Neurology Service, Dana Children's Hospital, Tel Aviv Sourasky Medical Center, 6 Weizmann Dr, Tel Aviv 64239, Israel. Email: bassan@post.tau.ac.il).

COMMENT. Israeli neurologists and neonatologists agree on initial management, but differ on treatment of intractable neonatal seizures, the harmfulness of neonatal seizures on developing brain, and need to monitor subclinical seizure activity. "Don't know" was a frequent answer by both specialties to questions regarding harmfulness of electrographic seizures and the need to treat them. Controversies in the literature need further research and answers.

Lamotrigine for partial seizures in patients aged 1 to 24 months was well tolerated and may be effective as adjunctive therapy, as shown by a randomized, double-blind, placebocontrolled study in 19 patients at Vanderbilt University, Nashville, TN (Pina-Garza, Levisohn P, Gucuyener K, et al. Neurology May 27, 2008;70:2099-2108). Rash occurred in $15 \%$ during the open label phase; none was Stevens-Johnson syndrome or toxic epidermal necrolysis. Children $<2$ years of age are considered therapeutic orphans since antiepileptic drug trials are hampered by multiple restrictions. This study demonstrates that drug trials at this age can be completed. (Goodkin HP, Buck ML. Editorial. Neurology 2008;70:20932094).

\section{INFECTIOUS DISORDERS}

\section{MATERNAL INFECTION AND RISK OF EPILEPSY IN CHILDHOOD}

The association between prenatal exposure to maternal specific infections during pregnancy and the subsequent risk of epilepsy in childhood was estimated in a prospective population-based birth cohort in Denmark followed for 8 years at University of Aarhus, Denmark. Of 90619 singletons, 646 children were identified with a diagnosis of epilepsy in the follow-up period. Children exposed to maternal cystitis in each trimester, pyelonephritis, 